\title{
A MULTIWINDOW TIME-FREQUENCY APPROACH BASED ON THE CONCEPTS OF ROBUST ESTIMATE THEORY
}

\author{
Irena Orović ${ }^{1}$, Nikola Žarić ${ }^{1}$, Srdjan Stankovic ${ }^{1}$, Moeness Amin ${ }^{2}$ \\ ${ }^{1}$ Faculty of Electrical Engineering, University of Montenegro, 81000 Podgorica, Montenegro \\ ${ }^{2}$ University of Villanova, Villanova, PA 19085
}

\begin{abstract}
An approach to multiwindow time-frequency analysis that provides robust performance in noisy environment is proposed. The concept of robust estimates of instantaneous frequency is used to define the optimal weighting coefficients for the multiwindow spectrogram. The proposed form of multiwindow spectrogram provides improved instantaneous frequency estimation for nonstationary signals in the presence of additive Gaussian noise. The efficiency of the proposed approach is tested in the experiments.
\end{abstract}

Index Terms - time-frequency analysis, instantaneous frequency estimation, Hermite functions, multiwindow approach

\section{INTRODUCTION}

Time-frequency analysis has been used in applications where the information, carried by the signal, is contained in its time-varying frequency content. Various time-frequency distributions have been proposed for the instantaneous frequency (IF) estimation [1]-[4]. One of the commonly used quadratic distributions is the spectrogram, defined as the square module of the short-time Fourier transform. It is simple for realization, and thus suitable for practical applications. However, the spectrogram has a low timefrequency resolution. In order to improve concentration in the time-frequency plane, other quadratic distributions are considered, such as the Wigner distribution, the S-method, etc. For instance, the Wigner distribution provides ideal representation when IF is a linear function of time. For nonlinear IF, the estimation accuracy can be improved by using the multiwindow approaches [5]-[8]. The multiwindow spectrogram [5] is defined as a weighted sum of spectrograms calculated with Hermite functions of different order. The weighting coefficients for the Hermite windowbased spectrograms are typically set according to desired constraints related to signal power localization in the timefrequency domain.

The signals used in the time-frequency analysis are usually, more or less, corrupted by the noise, which consequently affects the time-frequency representation. In order to reduce the noise influence, in this paper we propose a modified form of multiwindow spectrogram. The modification is related to the weighting coefficients, which are derived by using the concept of robust estimates [9]. The proposed modified form of multiwindow spectrogram provides good time-frequency representation and high accuracy of IF estimation even for low signal to noise ratio. It outperforms other standard distributions, including the former multiwindow spectrogram form.

The paper is organized as follows. Section II presents the theoretical background related to the standard form of multiwindow spectrogram. The multiwindow spectrogram based on the optimal weighting coefficients in noisy conditions is proposed in Section III. The efficiency of the proposed approach is tested experimentally in Section IV. Concluding remarks are given in Section V.

\section{THEORETICAL BACKGROUND - MULTIWINDOW TIME-FREQUENCY ANALYSIS}

\subsection{Optimal windows selection}

In order to improve the analysis of stationary and nonstationary spectrum over the standard methods, various multiwindow approaches have been introduced [5]-[7], [10][11]. In stationary spectrum estimation, the multiple windows are usually chosen as discrete prolate spheroidal sequences (DPSS) [10], being a family of orthonormal functions that maximize the energy in the given frequency band. Further, the minimum bias windows, having close behavior to the DPSS, have been used in spatial filtering for beamspace processing [11]. However, in the non-stationary cases, it is preferred to use functions that are optimally concentrated in the time-frequency domain with elliptic symmetry [12]. Such functions are orthogonal Hermite functions. They minimize the variance and simultaneously provide low bias estimate. The $k$-th order Hermite function is defined as:

$$
h_{k}(t)=\frac{1}{\sqrt{\sqrt{\pi} 2^{k} k !}} e^{-t^{2} / 2}(-1)^{k} e^{t^{2}}\left(\frac{d}{d t}\right)^{k} e^{-t^{2}}
$$


The recursive realization of the Hermite functions, which is considered attractive in practical applications, is given by:

$$
\begin{aligned}
& h_{0}(t)=\frac{1}{\sqrt[4]{\pi}} e^{-t^{2} / 2}, \quad h_{1}(t)=\frac{\sqrt{2} t}{\sqrt[4]{\pi}} e^{-t^{2} / 2}, \\
& h_{k}(t)=t \sqrt{\frac{2}{k}} h_{k-1}(t)-\sqrt{\frac{k-1}{k}} h_{k-2}(t), \quad \forall k \geq 2 .
\end{aligned}
$$

\subsection{Multiwindow spectrogram}

The multiwindow spectrogram has been introduced as an improved quadratic time-frequency distribution for analyzing signals with time-varying spectra. It has been defined as a weighted sum of spectrograms calculated for different window functions. By using the Hermite functions, as an optimal choice, the multiwindow spectrogram is defined as [5]:

$$
\operatorname{MWS}(t, \omega)=\sum_{k=0}^{K-1} d_{k}(t) S_{k}(t, \omega),
$$

where $S_{k}(t, \omega)$ represents a spectrogram calculated using the $k$-th order Hermite function as follows:

$$
S_{k}(t, \omega)=\left|\frac{1}{2 \pi} \int s(t) h_{k}(t-\tau) e^{-j \omega \tau} d \tau\right|^{2}
$$

Let us observe the signal in the form:

$$
s(t)=A(t) e^{j \varphi(t)}
$$

(5) This method is asymptotically unbiased in the noise-free

$A(t)$ is signal's amplitude, while $\varphi(t)$ is a pt function. Then, the conditional mean frequency obtained by using the spectrogram $S_{k}(t, \omega)$ is defined as [5]:

$$
\left\langle\omega_{k}\right\rangle_{t}=\frac{\int \omega S_{k}(t, \omega) d \omega}{\int S_{k}(t, \omega) d \omega}=\frac{\int A^{2}(t+\tau) h_{k}^{2}(\tau) \varphi^{\prime}(t+\tau) d \tau}{\int A^{2}(t+\tau) h_{k}^{2}(\tau) d \tau}
$$

Consequently, the conditional mean frequency $\langle\omega\rangle_{t}$ of the multiwindow spectrogram is given by:

$$
\langle\omega\rangle_{t}=\sum_{k=0}^{K-1} d_{k}(t)\left\langle\omega_{k}\right\rangle_{t}
$$

For signals with the $K$-th order polynomial phase:

$$
\varphi(t)=\sum_{n=0}^{K} a_{n} t^{n}
$$
case. However, as shown in [5], it outperforms standard distributions, such as the single window (standard) spectrogram, in noisy conditions as well.

the conditional mean frequency can be expressed as:

$$
\langle\omega\rangle_{t}=\sum_{k=0}^{K-1} \sum_{n=0}^{K-1} d_{k}(t) \frac{M_{n}^{k}(t)}{n !} \varphi^{(n+1)}(t),
$$

where $\varphi^{(n)}(t)$ denotes the $n$-th derivative of the phase, while the $n$-th order moment of the spectrogram is:

$$
M_{n}^{k}(t)=\frac{\int A^{2}(t+\tau) h_{k}^{2}(\tau) \tau^{n} d \tau}{\int A^{2}(t+\tau) h_{k}^{2}(\tau) d \tau}
$$

In order to recover the instantaneous frequency from (9), the following equation holds:

$$
\sum_{k=0}^{K-1} d_{k}(t) M_{n}^{k}(t)= \begin{cases}1 & n=0 \\ 0 & n>0\end{cases}
$$

Hence, to satisfy the instantaneous frequency constraints, the weights $d_{k}(t)$ are obtained by using at least $K$ spectrograms.

Furthermore, as it is derived in [5], a number of $2 K-1$ windows is required to satisfy the instantaneous bandwidth requirements. It means that, to satisfy both the instantaneous frequency and instantaneous bandwidth requirements, the weights are calculated by using $2 K-1$ windows:

$$
\sum_{k=0}^{2 K-2} d_{k}(t) M_{n}^{k}(t)= \begin{cases}1 & n=0 \\ 0 & n>0\end{cases}
$$

\section{OPTIMAL WEIGHTING COEFFICIENTS FOR NOISY ENVIRONMENT}

The peak frequency at each time is generally less affected by noise than the conditional mean frequency. Thus, in order to increase the performance of multiwindow distribution in the presence of noise, the concept of robust estimates is used in the sequel. Instead of conditional mean frequency, one may use the M-estimate of the instantaneous frequency [9]. It is obtained as a solution of the optimization problem given by:

$$
\widehat{\omega_{k}}=\arg \min _{\omega} J(\omega)
$$

where,

$$
J(\omega)=\int h(\tau)\left(F\left(e_{1}\right)+F\left(e_{2}\right)\right) d \tau
$$


The function $F$ is convex non-negative loss function, while $e_{1}$ and $e_{2}$ are, respectively, real and imaginary part of error function [9].

In order to determine optimal weights in the presence of noise, we may consider $\arg \min J(\omega)=S_{k}(t, \omega)$. The necessary minimum conditions in (13) and (14) can be given by the following complex valued equations:

$$
\begin{aligned}
& \frac{\partial J}{\partial \omega}=\int h(\tau)\left(\frac{\partial F\left(e_{1}\right)}{\partial \omega}+\frac{\partial F\left(e_{2}\right)}{\partial \omega}\right)=0, \\
& \frac{\partial F\left(e_{1}\right)}{\partial \omega}=F^{(1)}\left(e_{1}\right) \frac{\partial e_{1}}{\partial \omega}, \frac{\partial F\left(e_{2}\right)}{\partial \omega}=F^{(1)}\left(e_{2}\right) \frac{\partial e_{2}}{\partial \omega}
\end{aligned}
$$

By applying the linearization with respect to small IF bias $\Delta \omega_{k}$ and small influence of higher phase derivatives [9]:

$$
\delta \varphi(\tau)=\sum_{n=2}^{\infty} \varphi^{(n)}(t) \frac{\tau^{n}}{n !},
$$

the bias for the spectrogram with the $k$-th order window function is defined as:

$$
E\left\{\Delta \omega_{k}\right\}=\frac{\int A^{2}(t+\tau) h_{k}(\tau) \tau \delta \varphi(\tau) d \tau}{\int A^{2}(t+\tau) h_{k}(\tau) \tau^{2} d \tau} .
$$

Thus, the total bias for the multiwindow spectrogram with $K$ window functions can be defined in the form:

$$
E\{\Delta \omega\}=\sum_{k=0}^{K-1} c_{k}(t) E\left\{\Delta \omega_{k}\right\}
$$

where $c_{k}(t)$ are optimal weights for noisy conditions. Thus, by using (16) and (18), the total bias can be written as:

$$
E\{\Delta \omega\}=\sum_{n=2}^{\infty} \sum_{k=0}^{K-1} c_{k}(t) \frac{\int A^{2}(t+\tau) h_{k}(\tau) \tau^{n+1} d \tau}{\int A^{2}(t+\tau) h_{k}(\tau) \tau^{2} d \tau} \frac{\varphi^{(n)}(t)}{n !},
$$

Furthermore, the estimated instantaneous frequency can be written as follows:

$$
\omega(t)=\widehat{\omega}(t)+E\{\Delta \omega\}=\sum_{k=0}^{K-1} c_{k}(t) \sum_{n=1}^{\infty} \mathcal{H}_{n}^{k} \frac{\varphi^{(n)}(t)}{n !} .
$$

where $\mathcal{H}_{n}^{k}=\frac{\int A^{2}(t+\tau) h_{k}(\tau) \tau^{n+1} d \tau}{\int A^{2}(t+\tau) h_{k}(\tau) \tau^{2} d \tau}$ and $\mathcal{H}_{1}^{k}=1$ holds.

For the constant amplitude case we have: $H_{n}^{k}=\frac{\int h_{k}(\tau) \tau^{n+1} d \tau}{\int h_{k}(\tau) \tau^{2} d \tau}$

Finally, the weights $c_{k}$ can be obtained by solving the linear set of equations:

$$
\sum_{k=0}^{K-1} c_{k}(t) \mathcal{H}_{n}^{k}=\left\{\begin{array}{l}
1, n=1 \\
0, n>1
\end{array}\right.
$$

Based on the optimal weighting coefficients, the multiwindow spectrogram is defined as:

$$
\operatorname{MWS}(t, \omega)=\sum_{k=0}^{K-1} c_{k}(t)\left|\frac{1}{2 \pi} \int s(t) h_{k}(t-\tau) e^{-j \omega \tau} d \tau\right|^{2}
$$

Note that the windows $h_{k}$ should satisfy a number of conditions [9], e.g., they should be even and non-negative functions. Thus, we use the windows in the form: $h_{k}(t)=e^{-t^{2} / 4}\left|h_{k}(t)\right|$, where $h_{k}(t)$ is the $k$-th order Hermite function. Although, there are some of the required conditions that are not satisfied, the proposed form of windows, combined with the coefficients $c_{k}$, provides improved results in comparison with the former multiwindow approach. The optimal windows choice can be a topic for some further research.

\section{EXPERIMENTAL RESULTS}

Consider the noisy signal in the form:

$$
x(t)=e^{j(5 \cos (5.5 \pi t)-5 \sin (2.5 \pi t))}+v(t)
$$

where $v(t)=v_{1}(t)+j v_{2}(t), v_{1}(t)$ and $v_{2}(t)$ are Gaussian noises (zero mean with variance equal to 1 ). The time interval $t \in[-1,1]$, with sampling rate $T=2 / 128$, is used. The spectrogram, the Wigner distribution and the S-method are shown in Fig 1.a-c, respectively. Note that these distributions suffer from the noise and interference terms and do not provide satisfactory results. Further, the standard multiwindow spectrogram (MWS) is calculated with weights $d_{k}$ (Fig 1.d). The proposed multiwindow spectrogram with coefficients $c_{k}$ is shown in Fig 1.e. Three Hermite functions are used $(K=3)$. Namely, it has been shown experimentally that this number of functions provides efficient results. Clearly, the proposed approach with optimal weighting coefficients $c_{k}$, provides the best results for the considered noisy signal.

Based on the distributions from Fig 1, the IF is estimated for different values of SNR. The mean square errors (MSE) of IF estimations are calculated and presented in Table 1. The results are given for the spectrogram (SPEC) with rectangular window, the Wigner distribution (WD), the multiwindow spectrogram with coefficients $d_{k}$ (MWS_d, for $K=1$ and $K=3$ ), and the proposed multiwindow spectrogram MWS_c (for $K=1$ and $K=3$ ). The case with $K=1$ (one window function) is considered only for the comparison purpose. It can be observed that the proposed approach (MWS_c, $K=3$ ) provides high accuracy of IF estimation for signals with fast varying IF, even for low values of SNR. 


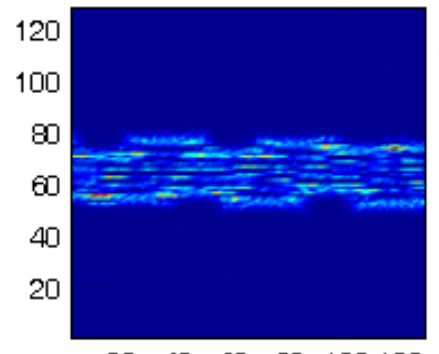

$20 \quad 40 \quad 60 \quad 80 \quad 100120$

a)

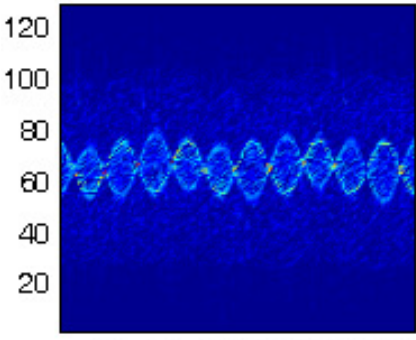

$20 \quad 40 \quad 60 \quad 80 \quad 100120$ b)

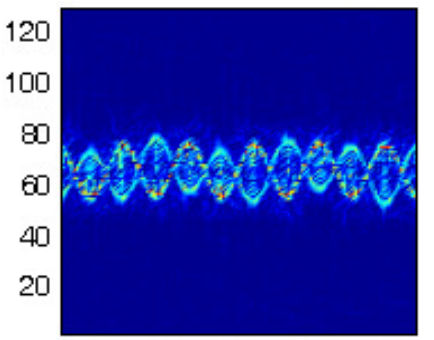

$20 \quad 40 \quad 60 \quad 80 \quad 100120$

c)

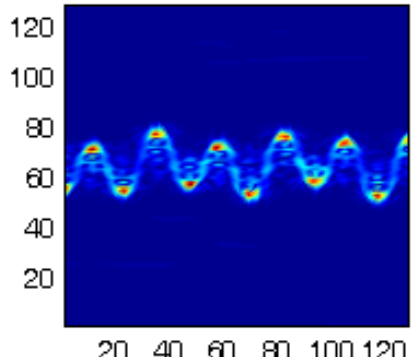

$20 \quad 40 \quad 60 \quad 80 \quad 100120$

d)

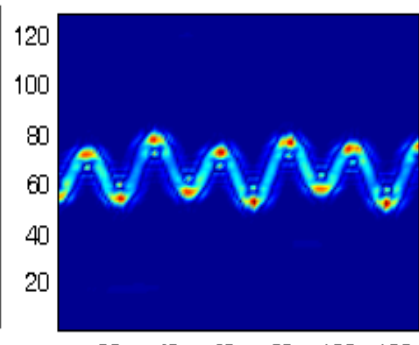

$\begin{array}{llllll}20 & 40 & 60 & 80 & 100 & 120\end{array}$

e)

Fig 1. a) spectrogram, b) Wigner distribution, c) S-method, d) multiwindow spectrogram with coefficients $d_{k}$, e) multiwindow spectrogram with coefficients $c_{k} . \mathrm{SNR}=10 \mathrm{~dB}$

Table 1. MSE for different distributions

\begin{tabular}{|l|c|c|c|}
\hline Distribution & SNR=5dB & SNR=10dB & SNR=15dB \\
\hline SPEC & 210 & 207 & 200 \\
\hline WD & 158 & 150 & 140 \\
\hline MWS_d, K=1 & 12.4 & 11.2 & 10.4 \\
\hline MWS_c, K=1 & 6.7 & 6.1 & 5.8 \\
\hline MWS_d, K=3 & 5.8 & 3.7 & 3 \\
\hline MWS_c, K=3 & 3 & 2 & 1.6 \\
\hline
\end{tabular}

\section{REFERENCES}

[1] B. Boashash, Time-Frequency Analysis and Processing, Elsevier, 2003, Amsterdam

[2] B. Boashash, "Estimating and interpreting the instantaneous frequency of a signal-Part 1: Fundamentals", Proc. of the IEEE, vol. 80, no. 4, pp. 520-538, 1992.

[3] J. Lerga and V. Sucic, "Nonlinear IF Estimation Based on the Pseudo WVD Adapted Using the Improved Sliding Pairwise ICI Rule," IEEE Signal Processing Letters, vol.16, no.11, pp.953-956, 2009.

[4] M. Jabloun, F. Leonard, M. Vieira, N. Martin, “A New Flexible Approach to Estimate the IA and IF of Nonstationary Signals of Long-Time Duration," IEEE Trans. on Signal Processing, vol. 55, no.7, pp: 3633 - 3644, July 2007

[5] F. Cakrak and P.J. Loughlin, "Multiwindow Time-Varying Spectrum with Instantaneous Bandwidth and Frequency
Constraints," IEEE Trans. on Signal Proc., vol. 49, no. 8, pp.1656-1666, Aug. 2001.

[6] G. Frazer and B. Boashash, "Multiple window spectrogram and time-frequency distributions," in Proc. IEEE ICASSP, vol. 4, 1994, pp. 193-296.

[7] M. Bayram and R. G. Baraniuk, "Multiple Window TimeFrequency Analysis," Proc. of the IEEE Signal Proc. International Symposium on Time-Frequency and Time-Scale Analysis, 1996, pp.173-176.

[8] I. Orovic, S. Stankovic, T. Thayaparan, LJ. Stankovic, "Multiwindow S-method for Instantantaneous Frequency Estimation and its Application in Radar Signal Analysis," IET Signal Processing, vol. 4, no. 4, pp.363-370, 2010.

[9] V. Katkovinik, "Robust M-estimates of the frequency and amplitude of a complex-valued harmonic," Signal Processing, vol. 77, no. 1, pp. 71-84, Aug. 1999.

[10] D. B. Percival and A. T. Walden, Spectral Analysis for the Physical Science, Cambridge, U.K., Cambridge University Press, 1993.

[11] M. Amin and N. Bhalla, "Minimum Bias Spatial Filters for Beamspace Direction-of-Arrival Estimation," Journal of Franklin Institute, vol. 335 B, no. 1, pp. 3552, Jan. 1998.

[12] J. Xiao and P. Flandrin, "Multitaper Time-Frequency Reassignment for Nonstationary Spectrum Estimation and Chirp Enhancement," IEEE Trans. on Signal Processing, vol. 55, no. 6, pp. 2851-2860, June 2007. 